\title{
USE OF ARTIFICIAL NEURAL NETWORKS TO PREDICT TERRITORIAL ECONOMIC INDICATORS
}

\section{GOCHA UGULAVA}

\section{PhD student}

Kutaisi University, Georgia

gochaugulava@gmail.com

For citation: Ugulava, G. (2019). Use of Artificial Neural Networks to Predict Territorial Economic Indicators, Globalization And Business, №8, pp. 143-146. https://doi.org/10.35945/gb.2019.08.019

Modern economic science is unthinkable without predicting and planning the prospects for economic life development. There is no doubt that forecasting has become one of the crucial scientific factors as the formulation public, socio-economic development strategy and tactics. In the notion of prognosis, we consider a scientifically reasoned discussion of the possible future states of the object under consideration. Economic Forecasting - is the process of developing economic forecasts based on the combination of scientific methods to understand economic events and their application in practice. There are many different mathematical and statistical tools in the arsenal of scientists as well as practitioners and economists today in purpose of forecasting. Modern advances in econometrics and forecasting increasingly allow scientifically grounded, objective and adequate forecasting to operate. However, the search for new methodological approaches to mathematical modeling complex systems continues. With the advent of artificial intelligence, intractable abundance and inexact logic, genetic algorithms and other modern research emerge new tools for data analysis and forecasting. To date, one of the most prominent effective tools for data analytics is artificial neural networks. Although there are attempts to forecast its financial markets, research into the potential use of this instrument in the economy is a contemporary issue.

Complete information about artificial neural networks can be found in many sources, for example the following literature: (Rojas, 1996; Kriesel, 2007; Russell, 2013; Simon, 2018). The article format does not allow us to discuss in detail the theoretical foundations for the construction and operation of these networks. We will provide general information on basic principles.

Artificial Neural Network - is a mathematical model created in the likeness of a human neural network, and its software and hardware implementation. Its constituent unit is an artificial neuron that imitates the properties of a biological neuron. (Fig. 1)

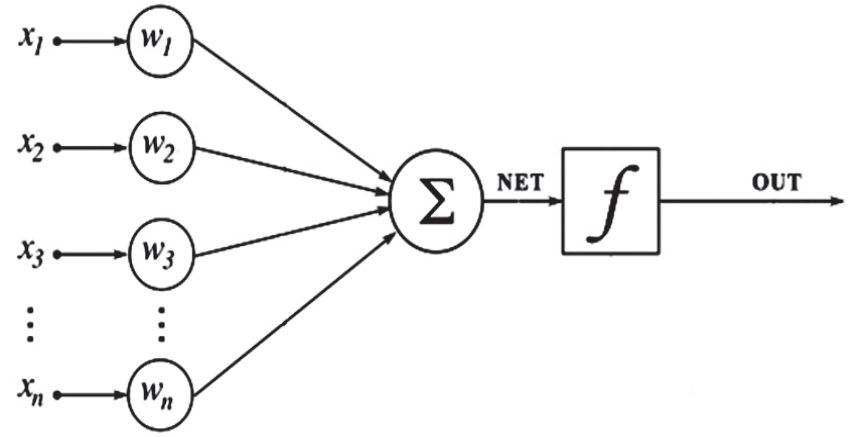

Fig. 1 Artificial Neuron Scheme

The input signals $(x 1, x 2, \ldots$, which are vector $X)$ are supplied at the input of an artificial neuron. Each signal is multiplied by the corresponding weight $(w 1, w 2, \ldots)$ and passed to the summing block $(\Sigma)$. The algebraic sum of the weighted input signals (output NET) is then transformed by the activation function (F) and finally gives the output neuron signal (OUT). Such neurons unite in layers. The set of neurons that make up the layer is a simple neural network. In network training process, it is achieved that the set of input signals corresponds to the desired output set. The training process is accomplished by a consistent supply of input vectors, with weight selection and optimization being carried out according to a defined procedure. In the training process, weights are gradually formed so that each input vector has a corresponding correct output vector. Deterministic and stochastic methods and algorithms are developed and used for network training. Depending on the specification of the model and the research task, one of the many available configurations (multilayer perceptron, networks with direct and back-propagation algorithms, Hopfield Network, Cohen-Grossberg Network, Hamming Network, Boltzmann Machine, etc) can be selected. (Stepanov, 2010: 5) 
In dynamic environments such as regional economies, where economic indicators base is scarce, and indicators themselves often do not meet the conditions of stationary and invariability of conditions, in a system dominated by mass processes, and complicated by difficulty of direct observations, where many interrelated factors operate, it is important to carry out applied research in order to introduce intelligent modeling technologies. These systems, events and processes, inherently, are stochastic, probabilistic in nature. Indeterminacy is their inner being. For the further development of the country, ensuring economic growth, its territorial development, plans for this development, adequate forecasting of key economic indicators are one of the most important components. Based on this, the purpose of this article is to study the possibilities of using artificial neural networks in these areas of economics by the example of analysis and forecasting of regional economic indicators.

We carried out modeling and forecasting of regional economic indicators using the artificial neural network of the three-layer perceptron architecture. The first layer is the input layer, which is supplied with 5 types of indicators, the second is the hidden layer - three-level with 14 neural nodes (8-4-2), the third is the output layer. The network architecture and neuron settings were automatically formatted through the programming language $R$ and its package - neuralnet (Fritsch, Guenther). (Fig. 2).

A number of socio-economic development indicators of Imereti region for the period 2006-2017 were used to train the network. The vectors are coded as follows: RGDP - Regional Gross Domestic Product, DFI - Foreign Direct Investment, FAI - Investment in Fixed Assets, EMP - Number of Employees, BT - Business Turnover, CPI - Consumer Price Index. All data are from the Imereti region and are taken from official GeoStat sources. (National Statistics Office of Georgia). All data was presented to the network as normalized. Data were normalized according to the following formula:

$$
Y=\frac{X-\min (X)}{\max (X)-\min (X)}
$$

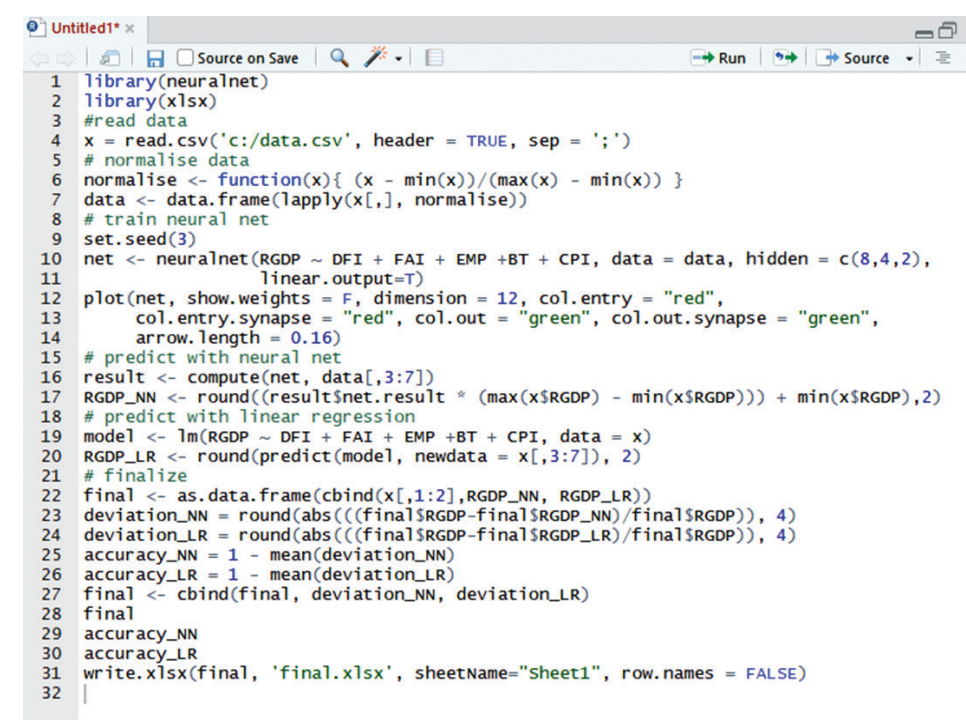

\begin{tabular}{|c|c|c|c|c|c|}
\hline YEAR & RGDP & RGDP_NN & RGDP_LR & $\begin{array}{c}\text { devia- } \\
\text { tion_NN }\end{array}$ & $\begin{array}{c}\text { devia- } \\
\text { tion_LR }\end{array}$ \\
\hline 2006 & 1474.54 & 1565.05 & 1565.16 & 0.0614 & 0.0615 \\
\hline 2007 & 1942.64 & 1890.92 & 1862.07 & 0.0266 & 0.0415 \\
\hline 2008 & 2347.64 & 2330.74 & 2267.65 & 0.0072 & 0.0341 \\
\hline 2009 & 2334.48 & 2347.93 & 2373.19 & 0.0058 & 0.0166 \\
\hline 2010 & 2561.54 & 2582.67 & 2606.00 & 0.0082 & 0.0174 \\
\hline 2011 & 2961.17 & 2925.86 & 2979.35 & 0.0119 & 0.0061 \\
\hline 2012 & 3091.43 & 3091.30 & 3095.24 & 0 & 0.0012 \\
\hline 2013 & 3070.41 & 3041.54 & 2991.89 & 0.0094 & 0.0256 \\
\hline 2014 & 3117.53 & 3201.87 & 3142.83 & 0.0271 & 0.0081 \\
\hline 2015 & 3397.89 & 3336.09 & 3379.95 & 0.0182 & 0.0053 \\
\hline 2016 & 3565.76 & 3578.14 & 3594.71 & 0.0035 & 0.0081 \\
\hline 2017 & 3984.60 & 3974.10 & 3991.57 & 0.0026 & 0.0017 \\
\hline
\end{tabular}

As a result of the program, an artificial neural network of the following structure was created (Fig. 3)

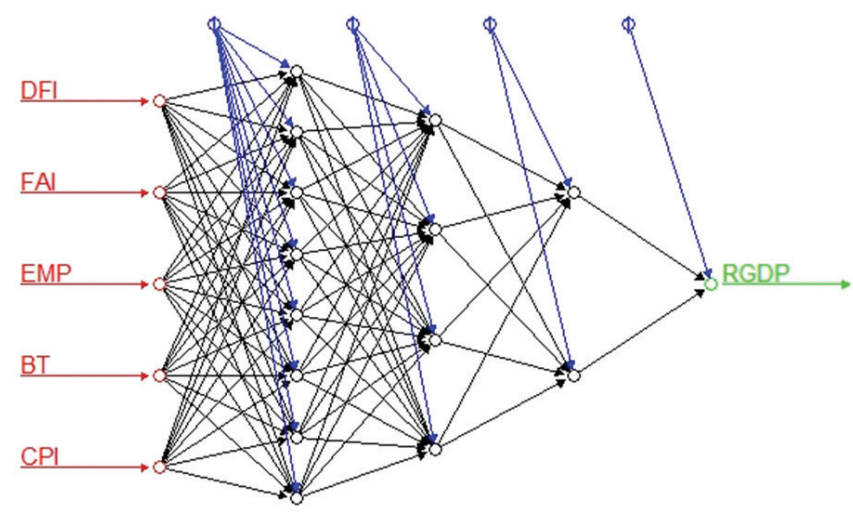

Error: 0.001989 Steps: 131

Fig. 3 Artificial Neural Network Structure

Then, during the forecasting phase, the data vectors were presented as data frame in five input parameters (DFI, $\mathrm{FAI}, \mathrm{EMP}, \mathrm{BT}, \mathrm{CPI}$ ), according to the neural network forecast of the regional gross domestic product (RGDP_NN) was calculated. Forecasting was done at the same time scale (2006-2017) to enable us to compare the predicted values with the actual ones to verify the level of forecast accuracy. We also tested the results of the neural network in another way - compared to the predicted values using multiple linear regression on the same data. Multiple linear regression method is an active method of the arsenal of economists and statisticians, characterized by high accuracy. The corresponding regression equation was calculated to build a model of the dependence as the dependent variable - RGDP on predictors - DFI, FAI, $\mathrm{EMP}, \mathrm{BT}, \mathrm{CPI}$. By it we obtained the linear regression forecast indicators of the regional GDP (RGDP_LR). The results of the program work are summarized in the table (Table).

Table. Determination of the accuracy of forecast indicators

\section{Fig.2 R Program Listing}


The accuracy of the predicted values calculated by the neural network was quite high (accuracy_NN $=0.9848417$ ), which was not declining but slightly ahead of the accuracy coefficients of the predicted values obtained through linear regression (accuracy_LR $=0.9810667$ ). Also, the predictive values calculated by the neural network with high adequacy and accuracy were compared with actual, existing ones. (Average deviation from actual $-1.5 \%$, smallest deviation - full accuracy coincidence, highest deviation - 6\%). Such high efficiency of the forecasting rates obtained through the network can be partly explained by the unity of network training and forecasting data, what was caused by overfitting of the network. However, based on the nature of the issue and the representativeness of the sample, it is unlikely that there are a large volume of random patterns that have a significant impact. It will be interesting to analyze other, including more comprehensive regional territorial indices with the above methodology. Under a larger sample, it is possible to train the model and then forecast using different indicators, which can eliminate the problem of overfitting of the network. Even under these conditions, as the research shows, the values of the prediction efficiency and determinant coefficients cannot be greatly reduced and the forecast fully satisfies the reliability and adequacy requirements. It is also a matter of further research to analyze the economic indicators presented as time series by the neural networks.

The magnitude of the deviation from the actual figures of the forecast and the accuracy of the calculations were obtained by the following formulas:

$$
\begin{gathered}
\text { deviation }_{N N}=\frac{\left|R G D P-R G D P_{N N}\right|}{R G D P} \text { deviation }_{L R}=\frac{\left|R G D P-R G D P_{L R}\right|}{R G D P} \\
\text { accuracy }_{N N}=1-\frac{1}{n} \sum \text { deviation }_{N N} \text { accuracy }_{L R}=1-\frac{1}{n} \sum \text { deviation }_{L R}
\end{gathered}
$$

Thus, the presented material shows that the use of artificial neural networks for the prediction of territorial economic indicators is reasonable and justified. Their role in analyzing and predicting indicators that are characterized by non-stationarity, dynamism, lack of a definite trend, periodicity, nonlinear structure is especially increased. It is therefore advisable to apply this method in regional economic studies, in predicting territorial development plans, strategies, targets and indicators. However, there are also a number of limitations to the use of neural networks. Artificial neural networks can be embedded in the methodological and instrumental arsenal of researchers, practitioners, economists, statisticians, and data analysts.

\section{REFERENCES:}

1. Raul Rojas (Author), J. Feldman (1996). Neural Networks: A Systematic Introduction. Springer, 1 edition

2. David Kriesel (2007). A Brief Introduction to Neural Networks. available at http://www.dkriesel.com

3. Stuart Russell, Peter Norvig (2013). Artificial Intelligence: Pearson New International Edition: A Modern Approach. Pearson, 3 edition

4. Haykin Simon (2018). Neural networks. Full course. Williams

5. Stepanov M. F. (2010) - Artificial neural networks in planning and control tasks. Saratov, p. 5

6. National Statistics Office of Georgia - www.geostat.ge 


\section{USE OF ARTIFICIAL NEURAL NETWORKS TO PREDICT TERRITORIAL ECONOMIC INDICATORS}

\section{GOCHA UGULAVA}

\section{PhD student}

Kutaisi University, Georgia

gochaugulava@gmail.com

KEYWORDS: REGIONAL ECONOMICS, FORECASTING, ARTIFICIAL NEURAL NETWORKS, MATHEMATICAL MODELING, TERRITORIAL ECONOMIC INDICATORS.

For citation: Ugulava, G. (2019). Use of Artificial Neural Networks to Predict Territorial Economic Indicators, Globalization And Business, №8, pp. 143-146. https://doi.org/10.35945/gb.2019.08.019

\section{SUMMARY}

Modern economic science is unthinkable without predicting and planning the prospects for economic life development. There are many different mathematical and statistical tools in the arsenal of scientists as well as practitioners and economists today in purpose of forecasting. To date, one of the most prominent effective tools for data analytics is artificial neural networks. Artificial Neural Network - is a mathematical model created in the likeness of a human neural network, and its software and hardware implementation. We carried out modeling and forecasting of regional economic indicators using the artificial neural network of the three-layer perceptron architecture. The network architecture and neuron settings were automatically formatted through the programming language $\mathrm{R}$ and its package - Neuralnet. During the forecasting phase, the data vectors were presented as data frame in five input parameters (DFI, FAI, EMP, BT, CPI), according to the neural network forecast of the regional gross domestic product (RGDP_NN) was calculated. All data are from the Imereti region and are taken from official GeoStat sources. Forecasting was done at the same time scale (2006-2017) to enable us to compare the predicted values with the actual ones to verify the level of forecast accuracy. We also tested the results of the neural network in another way - compared to the predicted values using multiple linear regression on the same data. The accuracy of the predicted values calculated by the neural network was quite high, which was not declining but slightly ahead of the accuracy coefficients of the predicted values obtained through linear regression. Also, the predictive values calculated by the neural network with high adequacy and accuracy were compared with actual, existing ones.

Presented material shows that the use of artificial neural networks for the prediction of territorial economic indicators is reasonable and justified. Their role in analyzing and predicting indicators that are characterized by non-stationarity, dynamism, lack of a definite trend, periodicity, nonlinear structure is especially increased. It is therefore advisable to apply this method in regional economic studies, in predicting territorial development plans, strategies, targets and indicators. 